\title{
FActores Que Influyen En LA Elaboración Del Proyecto De Tesis De Maestría
}

\author{
Factors that influence the development of the Magister's Thesis Project
}

\author{
Renatto Merino Solari ${ }^{1}$
}

\begin{abstract}
RESUMEN
El texto tiene su origen en el análisis crítico y reflexivo de mi ejercicio profesional como docente de asignaturas de tesis en diferentes programas de maestría. El propósito es explorar los factores que influyen en la elaboración del proyecto de tesis de maestría; asimismo, comprender la forma en que se manifiestan durante esta fase. Se sustenta que los factores intervinientes son diversos e interactúan formando contextos que facilitan o dificultan la experiencia particular del tesista.
\end{abstract}

Palabras claves: Proyecto, tesis, maestría, investigación, factores, docencia, educación.

\section{Abstract}

The text has its origin in the critical and reflective analysis of my professional practice as a professor of thesis subjects in different magister's programs. The purpose is to explore the factors that influence the development of the magister thesis project; also, to understand the way they manifest during this phase. It is based that intervening factors are diverse and interact forming contexts that facilitate or hinder the particular experience of the student.

Keywords: project, magister, thesis, research, factors, teaching, education.

\section{I}

a elaboración de la tesis es una de las experiencias más difíciles que deben enfrentar los estudiantes para alcanzar la meta de graduarse. La situación alcanza tal magnitud que autores como Abreu (1), Carlino (2) y Ferrer y Malaver (3) se refieren al síndrome de "todo menos tesis". Esta idea define a la etapa en la cual el tesista ha culminado sus estudios cumpliendo satisfactoriamente con el plan de estudios y los créditos exigidos por el programa y, por tanto, debe desarrollar la investigación y elaborar la tesis para su sustentación; sin embargo, el período se prolonga más de lo esperado postergándose indefinidamente la culminación del proceso. Esta realidad es un fenómeno relativamente común en las universidades latinoamericanas. Entre sus consecuencias destaca el incremento de las tasas de no graduados que evidencian los programas de postgrado. Los factores que explican este problema resultan de la interacción entre aspectos personales e institucionales.

Entre otros aspectos, el tiempo que toma realizar una investigación es un factor determinante en eléxito o fracaso de la empresa. La realización de una tesis es un proceso largo que presenta diferentes etapas y situaciones. Se considera que son dos los momentos de mayor complejidad para el novel investigador: la fase inicial, cuando se propone la investigación, y

1 Magíster en Antropología. Docente de la Facultad de Educación, Universidad Peruana Cayetano Heredia. 
la fase final, cuando lo trabajado toma forma en un informe final para su presentación ante un público especializado en el tema.

En esta ocasión, el interés estará centrado en la fase inicial de la tesis, ya que este es el momento en el cual el estudiante de la maestría propone, planificay proyectaunainvestigación relevante, que deberá cumplir con las condiciones académicas y administrativas establecidas por el programa de postgrado. Como señala Arnoux et al. (4), esta es una etapa importante de la investigación, pues diseñar un proyecto de tesis plantea dificultades propias.

El proyecto es el primer documento que escribe un tesista para escrutinio público; es decir, para una evaluación más allá de los límites de una asignatura y del marco de revisión del docente. Según Arnáez, el proyecto es un escrito que expresa un "conjunto de premisas relacionadas con la especificidad de una esfera social determinada (estudios de postgrado); con una temática correspondiente al área de conocimiento...; con una estructura aceptada por la comunidad académica universitaria y con la valoración de los potenciales lectores..."

(5). Asimismo, "implica un quehacer interdisciplinario al tener que conjugar el saber lingüístico y el saber específico sobre el que versa el proyecto" (5). Por tanto, se trata de un texto complejo que demanda de su autor el conocimiento y manejo de las formalidades de la escritura académica, así como de las habilidades de un experto para tomar decisiones. En este sentido, lo que pretendemos es explorar algunos de los factores que influyen durante la elaboración del proyecto de tesis de maestría; y, también se busca comprender la forma en que estos factores interactúan en la experiencia del tesista.

Este escrito se origina en mi experiencia como docente de asignaturas de investigación en diferentes programas de postgrado (Humanidades, Ciencias sociales y Educación) de universidades tanto del sector público como privado. Durante varios años he tenido la oportunidad de dirigir los cursos iniciales de investigación cuyo logro era proponer un proyecto de investigación para la tesis. Los insumos principales de esta indagación han sido los apuntes de las sesiones de clase basados en las observaciones y en los diálogos sostenidos con los estudiantes. Los apuntes corresponden a diferentes contextos y situaciones. El análisis de estos materiales generó las primeras reflexiones sobre el problema y permitieron identificar un conjunto de factores intervinientes. Esta información inicial fue enriquecida conversando sobre el tema con egresados de diferentes programas de maestría pero de las mismas áreas del conocimiento. De manera concomitante, el análisis de la literatura especializada permitió un diálogo interactivo entre mi experiencia práctica y los aportes conceptuales.

\section{II}

El tema de la elaboración de la tesis en el postgrado ha generado una importante bibliografía. Aquí reseñaremos algunos de los trabajos realizados tomando como eje a los factores que influyen durante la investigación. Los estudios muestran que en el desarrollo de la tesis actúan factores tanto personales como institucionales, y su interacción puede favorecer $y / 0$ dificultar la experiencia del tesista. Así, para Carlino (2), los factores forman contextos que facilitan la producción de una tesis, como por ejemplo: pertenecer a un grupo con un programa de investigación, estar dedicado a investigar a tiempo completo, contar con una beca y recursos. Por otra parte, también existen contextos que obstaculizan: la falta de equipo, la incertidumbre de no saber si culminará la investigación, investigar a tiempo 
parcial, el sentimiento de soledad y la idea de conflicto interno.

En un trabajo posterior, Reisin y Carlino (6) exploraron los factores que intervienen en la culminación de una maestría; en el caso de la realización de la tesis, entre los factores personales destacan: el apoyo de la familia, el tiempo dedicado al trabajo, la motivación por una mejor inserción laboral y/o una redefinición identitaria profesional; mientras que entre los factores institucionales resaltan: el soporte administrativo de la institución en cuanto a plazos, procesos y espacios es relevante para la culminación de la tesis, así como el soporte pedagógico-didáctico pues las tesis asumidas de forma individual y solitaria no suelen prosperar; $\mathrm{y}$, otro aspecto importante es el acceso a becas, sin embargo, es visto como un factor relativo. La interacción entre el nivel personal y el institucional también son planteadas por Ambrosini y Mombrú (7) quienes encuentran que los elementos diferenciadores que caracterizan a las personas que logran culminar la tesis son la contención institucional, la capacitación del personal que asesora y acompaña el proceso de investigación y la disposición personal del tesista. En el trabajo de Ochoa (8), acerca de las dificultades y retos que implica realizar una tesis, se afirma que todo el proceso de investigación resulta complejo para el tesista pero son los momentos iniciales (delimitación del tema, el estado de la cuestión, el marco teórico y el diseño metodológico) y la fase final (redacción del informe) los que plantean los mayores retos y dificultades.

Por otra parte, Ochoa y Cueva (9) se ocupan del bloqueo durante la elaboración de la tesis de maestría. Sus resultados muestran que las causas de este fenómeno son variadas y están relacionadas con aspectos personales, académicos e institucionales. Entre las causas del bloqueo encontramos factores coincidentes con los mencionados en los estudios anteriores. Así por ejemplo: la falta de tiempo, la presión del tiempo y los compromisos laborales son consideradas las causas principales del bloqueo. Otros factores también coincidentes son el desconocimiento del tema, del proceso de investigación y la relación con el asesor.

En cuanto al proyecto de tesis existe una menor cantidad de investigaciones. Los textos revisados lo han abordado desde las dificultades presentadas por los estudiantes para su preparación. Así, Perdomo y Flores (10) encontraron que predominaban los problemas relacionados con la redacción científica, especialmente al momento de plantear el problema y justificarlo, en el uso del sistema de referencia y en la redacción de los antecedentes; asimismo, se evidenciaban dificultades con el manejo del discurso argumentativo y en la definición de los aspectos metodológicos de la investigación. Blanco et al. (11) obtuvieron resultados muy similares, pues los principales problemas estaban en la redacción y la formulación del problema, los objetivos, la metodología y las referencias bibliográficas, además del presupuesto. Grasso (12) describe las dificultades que se presentan en la redacción de cada una de las partes del proyecto; y, sostiene que elaborar un proyecto es una tarea de ensayo y error, de organización y revisión constante de diversos elementos con los que el estudiante no siempre se encuentra familiarizado. Desde una perspectiva distinta, Arnáez (5) aborda el proyecto de investigación como un género discursivo analizando las características textuales de sus componentes; así, el autor concluye que se trata de un texto complejo que demanda del tesista saberes interdisciplinarios.

De la revisión bibliográfica planteamos lo siguiente: los factores influyentes son diversos 
y contextuales; es decir, tienen diversas procedencias e interactúan configurando escenarios que coadyuvan y/o dificultan la experiencia particular del tesista. De acuerdo con Carlino (2), los contextos que facilitan / obstaculizan la escritura de una tesis están vinculados a condiciones institucionales y personales específicas. Los contextos no son favorables o desfavorables a priori ni atributos específicos de alguno de los actores. Se trata de circunstancias muy concretas que definen el rol que juega cada uno en la experiencia particular del tesista. Sin embargo, los estudiantes suelen percibir los obstáculos de forma individual, como si fueran atributos personales que ellos llevan consigo.

\section{III}

Veamos cómo se manifiestan un conjunto de factores identificados durante mi experiencia docente.

"ES NEGATIVO ESTUDIAR Y TRABAJAR, SE DA PRIORIDAD AL TEMA LABORAL" (Luis, maestría en Educación)

El factor tiempo es uno de los más recurrentes cuando se conversa con los estudiantes acerca de las dificultades presentadas para el cumplimiento de los avances programados. Generalmente se refieren a dos situaciones: la falta de tiempo y su organización. La primera es la principal manifestación, pues los estudiantes son profesionales que trabajan, en la mayoría de los casos, jornadas laborales completas. Algunos tienen más de un compromiso laboral, otros, además de compartir el trabajo con la maestría, realizan estudios complementarios, como por ejemplo idiomas. La jornada laboral oscila entre las 40 y 48 horas de trabajo semanal; asimismo, sus centros de trabajo no brindan mayores incentivos ni facilidades para trabajar y estudiar en mejores condiciones. A esto debemos sumar las demandas del entorno familiar, pues se presentan casos de personas que tienen responsabilidades como padres y madres de familia. En este punto, la condición de género parece exigir más a las mujeres.

La organización del tiempo se encuentra asociada a su escasez, pero no siempre es así; existen casos en los que el estudiante dispone del tiempo necesario para sus estudios, sin embargo, expresan dificultades para organizarse. Algunos llegan a reconocer su falta de disciplina y constancia para el desarrollo de las tareas asumiéndolo como una falta de capacidad. La organización del tiempo la perciben como una responsabilidad personal a diferencia de la falta de tiempo que la ven como una presión externa. Al margen de las percepciones, investigar es una actividad que exige dedicación completa; de lo contrario, el abandono del proyecto se vuelve un riesgo permanente.

Otro aspecto relacionado al tiempo es la falta de sincronía entre los plazos administrativos y el tiempo de la investigación. La elaboración del protocolo de investigación suele exigir el cumplimiento de plazos. Este es un tiempo objetivo, de tipo burocrático, que busca cumplir metas; no es un tiempo que nazca de las necesidades de la investigación y del estudiante. La consecuencia de esta distemporalidad es el apresuramiento que vulnera la calidad del texto o su postergación, que en algunos casos lleva al abandono de la empresa.

“LA TESIS ES UN RETO" (Octavio, maestría en Psicología Educativa)

La motivación es un factor que opera positivamente y está presente en todos los estudiantes. Realizar un posgrado alimenta las expectativas profesionales, laborales, así como de mejora personal. En cuanto 
a la experiencia de investigar, a pesar de manifestar dudas y temores, la asumen con entusiasmo. Al ser profesionales en ejercicio suelen llegar al curso con temas de interés. Han identificado situaciones complejas en sus espacios laborales y los motiva la posibilidad de aportar a mejorar la realidad en la que se desenvuelven profesionalmente. Esto hace que las expectativas en el curso y en el docente sean elevadas, pues esperan las pautas apropiadas para tener claridad y dar sentido al proceso.

La realización dela tesis se convierteen una meta personal generándose una identificación muy particular con el tema propuesto. Evidencian gran interés por proponer un tema novedoso, innovador, que visibilice alguna realidad, que contribuya a resolver un problema o rinda tributo a un autor significativo. El interés que despierta el tema en el tesista es fundamental para sostener la motivación durante las diferentes etapas del largo proceso de la tesis. Además, la motivación permite mantener el ritmo de trabajo y evitar los "bajones", que suelen ser comunes por la inexperiencia del estudiante y por los retos de un proceso que demanda tiempo y paciencia.

En la fase inicial de investigación el planteamiento del problema es un momento particular. Pasar del tema a la problemática les demanda mucho esfuerzo. Existe la sensación de estar andando a tientas, sin rumbo fijo, de estar frente a retos para los que no se encuentran preparados, especialmente cuando descubren que la idea inicial no constituye un tema relevante y deben reorientarla. Los momentos de desánimo no son extraños en esta fase inicial; a ello se suma la falta de tiempo y la sobrecarga de responsabilidades (personales y académicas) configurándose un universo particular que podría tornarse crítico. Algunos estudiantes dejan de estudiar por motivos de salud. En determinadas circunstancias, el estrés y la ansiedad pueden ser inmanejables.

\section{"NO RECUERDO QUE ME HAYAN PREPARADO PARA ESTO" (Alfonso, maestría en Filosofía)}

Son pocos los estudiantes que han tenido experiencia investigando; de igual manera, carecen de adiestramiento en escritura y lectura académica. En los diálogos sostenidos se evidenció que esto era una herencia de la formación del pregrado: la mayoría se ha titulado recurriendo a alguna modalidad alternativa a la tesis. Ello es expresión, entre otros factores, de la poca atención que se le otorga a la formación en investigación en los planes de estudio de las carreras. Las asignaturas privilegian la transmisión de los contenidos disciplinares y la investigación queda reducida a la asignatura de metodología, durante los ciclos iniciales, y a los cursos de tesis, hacia el final de la carrera. Por otra parte, cuando se investiga en las asignaturas, las características metodológicas así como las formas de presentación de los productos finales son de una gran heterogeneidad. A ello debemos sumar el perfil de los docentes de investigación. Al parecer, oscilamos entre el reconocido investigador para quien enseñar resulta una actividad secundaria y el docente con poca o ninguna experiencia en investigación que enseña reproduciendo los manuales de metodología. Los primeros son la minoría.

Por el contrario, los estudiantes que elaboraron tesis en el pregrado valoran mucho esta experiencia y reconocen la utilidad que tiene para la maestría. Les ayuda a evitar o superar las dificultades que se van presentando. Entre otros aspectos, resaltan el haber desarrollado una metodología de trabajo, así como la familiarización con enfoques teóricos y metodológicos, el manejo de la bibliografía especializada, la escritura de acuerdo a las convenciones académicas $y$, especialmente, 
el haber vivenciado con éxito un proceso complejo. Estos estudiantes son los que encuentran el camino del proyecto más pronto y con menos dificultades. La utilidad de la experiencia de tesis la extienden hasta el conocimiento de los procesos administrativos, pues el registro del proyecto, la designación del asesor(a), el trámite para la sustentación y el posterior trámite para la obtención del grado académico resultan, en algunas universidades, un camino largo y ajeno.

\section{"EL PROYECTO PODRÍA SER UN TEXTO LIBRE, CON INTUICIONES BIEN PLANTEADAS" (Pedro, maestría en Filosofía)}

La estructura del proyecto de investigación expresa el monismo metodológico que predomina en la investigación científica. El proyecto y también el informe final corresponden a los pasos que propone la metodología positivista de investigación. Se trata de una estructura lineal y rígida que proviene de disciplinas como la biología y la física que debido a los importantes logros alcanzados durante los siglos XIX y XX se convirtieron en modelos disciplinares para otras áreas del conocimiento. No obstante, el monismo metodológico resulta problemático para formas de acercarse a la realidad que pueden ser muy diversas como ocurre en los campos de las humanidades, las ciencias sociales y la educación.

Si bien es cierto, en los últimos años la investigación cualitativa ha ganado espacios como forma válida de producción de conocimiento, el paradigma positivista continúa siendo hegemónico. $\mathrm{O}$, se duda del carácter científico del enfoque cualitativo o se acepta, pero subordinada a la lógica positivista de la investigación cuantitativa. Este proceso por su rigidez y linealidad es incapaz de acompañar la multiplicidad de formas particulares de conocer. Se trata de epistemologías y metodologías distintas, son dos lógicas de producción de conocimientos bien diferenciadas y cada una requiere de espacios propios pero también de espacios para intercambios simétricos.

En mi experiencia he visto constantemente las dificultades que tienen los estudiantes de maestría cuando pretenden plantear una investigación cualitativa. Como los protocolos responden a la lógica positivista, los formatos utilizados para los proyectos deben ser "traducidos" para su "adecuación" a las formas cualitativas de investigación. El resultado es una desnaturalización de la estructura del protocolo (la perspectiva del monismo metodológico) y también de la propuesta del estudiante, quien debe forzar sus ideas para que "encajen" en el documento. Este es un problema que vuelve a presentarse durante la elaboración del informe final.

\section{"ERAN CURSOS AISLADOS, PARTICULARES, NO RELACIONADOS AL TRABAJO DE INVESTIGACIÓN" (María, maestría en Psicología)}

El plan de estudio de una maestría está compuesto por un conjunto de asignaturas especializadas en función a los objetivos del programa. En lo que respecta a los cursos de tesis, y atendiendo a las características del programa, estos pueden oscilar entre dos y cuatro. Al margen de la cantidad, lo importante es cómo se tejen los planes de estudio. Aquí, el problema principal es la falta de articulación con respecto a la elaboración de la tesis, pues las asignaturas tienen sus contenidos disciplinares, metodologías y logros particulares. Los profesores orientan sus cursos en función de su experiencia profesional/académica y ello no siempre coincide con las necesidades de investigación 
del estudiante. Además, se dialoga poco entre los colegas y esto se expresa en el desarrollo de las clases. La demanda de cada asignatura en lecturas, tareas, indagaciones y productos es muy grande y no siempre están vinculadas con los intereses del tesista. En este escenario, son expresiones comunes de los estudiantes de la maestría: "Profesor, nos hemos olvidado del tema de la tesis", "El curso... es interesante pero nos absorbe mucho", "Los trabajos del profesor... nos quitan todo el tiempo".

En el caso de los estudiantes que logran vincular su tema de investigación con los contenidos de las otras asignaturas se produce un avance importante en la investigación. Sin embargo, esto es algo que sucede más como circunstancias particulares que como parte de un trabajo integrado e interdisciplinario. Es interesante resaltar que los estudiantes viven esta situación como una responsabilidad personal, que es deber de ellos hacer un esfuerzo mayor al que ya realizan y generar el tiempo necesario para la tarea de hacer la tesis

\section{“EL ASESOR FUE IMPORTANTE, APRENDÍ MUCHO" (Aldo, maestría en Antropología)}

El rol del asesor es fundamental durante todos los momentos de la elaboración de una tesis. En la fase inicial de la investigación es posible dos situaciones: 1) el asesor es designado al registrarse el proyecto de investigación y 2) se asigna un asesor para que participe desde la elaboración del protocolo de investigación. En el primer caso, la construcción de la propuesta de investigación la realiza el tesista prácticamente solo y su principal soporte son las pautas que recibe en los cursos de tesis. El rol del asesor se inicia luego de haber sido escrito el proyecto, es más un requisito administrativo que un acompañante de la propuesta. En el segundo caso, el asesor participa del diseño de la propuesta desde su concepción. Los estudiantes valoran positivamente la participación del asesor en esta fase inicial, pues su acompañamiento es fundamental en aquellas partes del proyecto que les genera mayores dificultades: delimitar el problema de investigación, elaborar el marco teórico, definir la metodología y la redacción del texto.

Los estudiantes esperan del asesor que sea un experto en el tema y también que realice un acompañamiento constante $y$ comprometido. De manera muy especial valoran la comprensión y la paciencia del asesor. La primera porque ayuda a "liberarlos" del sentimiento de responsabilidad/culpa que sienten por considerar (erróneamente) que son los únicos o principales responsables del proceso. La segunda, porque requieren que los esperen. En el aprendizaje cada uno tiene su tiempo y la lentitud es, en ocasiones, una necesidad.

\section{IV}

El texto se planteó explorar los factores que incidenenelproceso deelaboracióndelproyecto de tesis de maestría y comprender la forma en que se manifiestan. A partir de lo observado en el aula se identificaron los siguientes factores: el tiempo, la motivación, la experiencia previa del estudiante como investigador, la estructura del proyecto de investigación, la forma de articulación de las asignaturas en el plan de estudios y el rol del asesor. Algunos de estos factores tienen su origen en las características personales y académicas de los estudiantes; mientras que otros están relacionados con el rol de las instituciones y el soporte académico que brindan. En la práctica, la variedad y particularidad de las experiencias y situaciones entrelazan tanto las fuentes como las responsabilidades. Esto significa que los factores no se presentan de forma aislada, por 
el contrario, generan intersecciones y originan escenarios singulares que pueden coadyuvar o dificultar la tarea del tesista.

El proyecto de tesis es un texto entre académico y administrativo que demanda de su autor habilidades complejas. Investigar requiere estar inmerso en el mundo de las disciplinas científicas $\mathrm{y}$, especialmente, en sus formas de producción del conocimiento. En este sentido, proponer y planificar una investigación es una actividad compleja que demanda una preparación especial; sin embargo, el estudiante de la maestría suele tener poca experiencia en este campo, pues ha recibido una formación con énfasis en la práctica profesional, además de un ejercicio profesional que generalmente no le demanda producir conocimiento. En estas condiciones, plantear un tema de investigación y proyectar su desarrollo epistemológico y metodológico podría resultar una tarea llena de obstáculos y dificultades. Es como trazar el mapa de un camino que nunca se ha recorrido.

Para finalizar, un balance personal. En mi práctica docente había observado que la realización del proyecto constituía una actividad de mucha dificultad para la mayoría de los estudiantes. Asumía como explicación que los estudiantes acceden a la maestría con un conjunto de carencias académicas propias de su formación previa. Asimismo, y sin tener muy claro en qué condiciones, consideraba que estas limitaciones ya deberían estar superadas $\mathrm{y}$, por tanto, encontrarse preparados para enfrentar los retos del programa. En gran medida compartía el imaginario que pone las dificultades del proceso, principalmente, en el estudiante. En consecuencia, durante las clases me centraba en el cumplimiento de los contenidos buscando alcanzar los logros de la asignatura. En ocasiones parecía estar más preocupado en cumplir lo programado que en acompañar el ritmo tan singular de los estudiantes. El ejercicio de someter a un análisis crítico y reflexivo los supuestos de mi experiencia me permitió modificar las percepciones iniciales que tenía sobre el problema y reorientar el sentido de la praxis profesional.

\section{REFERENCIAS BibliográficAS}

1. Abreu J. Síndrome Todo Menos Tesis (TMT). Daena: International Journal of Good Conscience. [Internet]. 2015; 10(2): 246-259. URL disponible en: http:/ / www.spentamexico.org/ v10-n2 / A14.10(2)246-259.pdf [acceso 20/4/2018]

2. Carlino P. La experiencia de escribir una tesis: contextos que la vuelven más difícil. En: II Congreso Internacional Cátedra UNESCO Lectura y Escritura. Pontificia Universidad Católica de Valparaíso, 5-9 de mayo de 2003. [Internet] Valparaíso: Pontificia Universidad Católica de Valparaíso. URL disponible en: https:/ / media.utp.edu.co/referencias-bibliograficas / uploads / referencias / ponencia/239-la-experiencia-de-escribiruna-tesis-contextos-que-la-vuelven-m-s-difcilpdf-OGf01articulo.pdf [acceso 01/03/2018]

3. Ferrer de Valero Y, Malaver Hernández M. Factores que inciden en el Síndrome Todo Menos Tesis (TMT) en las maestrías de la Universidad del Zulia. Opción [Internet]. 2000; 31:112-129. URL disponible en: https://dialnet.unirioja.es/descarga/ articulo/2474955.pdf [acceso 18/04/2018]

4. Arnoux E, Borsinger A, Carlino P, Di Stefano M, Pereira, C y Silvestre A. La intervención pedagógica en el proceso de escritura de tesis de posgrado. Revista de la Maestría en Salud Pública. [Internet] 2005;6. URL disponible en: https://media. utp.edu.co/referencias-bibliograficas / uploads / referencias / articulo / 89-la-intervencin-pedaggica-en-el-proceso-deescritura-de-tesis-de-postgradopdf-0jI9S-articulo.pdf [acceso 21/04/2018]

5. Arnáez P. El proyecto de trabajo de grado: una experiencia discursiva universitaria. Zona Próxima [Internet]. 2014;20:127143. URL disponible en: http://rcientificas.uninorte.edu.co/ index.php/zona/article/viewArticle/ 6160 [acceso 05/03/2018]

6. Reisin S, Carlino P. Factores que favorecen u obstaculizan la finalización de una maestría en ciencias sociales. El punto de vista de los involucrados. En: I Congreso Internacional de Investigación y Práctica Profesional en Psicología, XVI Jornadas de Investigación en Psicología y Quinto Encuentro de Investigadores en Psicología del Mercosur. Psicología y Sociedad Contemporánea: Cambios Culturales. [Internet] 2009. Buenos Aires: Facultad de Psicología de la Universidad de Buenos Aires. URL disponible en: https: / / www.aacademica. org/paula.carlino/170 [acceso 23/04/2018]

7. Ambrosini C, Mombrú A. Bendita tesis: tribulaciones personales e institucionales. Perspectivas Metodológicas. [Internet] 2017;19(II). Disponible en: http://revistas.unla.edu.ar/ epistemologia/issue / view / 118 [acceso 22/04/2018]

8. Ochoa L. La elaboración de una tesis de maestría: exigencias y dificultades percibidas por sus protagonistas. Entornos. [Internet] 2011;24:171-183. URL disponible en: https: / / dialnet. 
unirioja.es $/$ servlet $/$ articulo?codigo $=3798839$ [acceso 22 $/ 04 / 2018$ ]

9. Ochoa L, Cueva A. EL bloqueo en el proceso de elaboración de una tesis de maestría: angustias y desazones percibidas por sus protagonistas. Lenguaje. [Internet] 2017; 45(1): 61-87. Disponible en: http:// revistalenguaje.univalle.edu.co/index. php/lenguaje/article/view / 4614 [acceso 11/05/2018]

10. Perdomo B, Flores M. Dificultades y errores más frecuentes en la elaboración de un proyecto de investigación. Caso: Estudiantes de Odontología - ULA. En: Memorias del Congreso Regional de Investigación y Pedagogía. Universidad Pedagógica Experimental Libertador. Instituto Pedagógico de Barquisimeto "Luis Beltrán Prieto Figueroa", 12-15 de mayo 2014 [Internet]. Barquisimeto: Universidad Pedagógica
Experimental Libertador. URL disponible en: http://www. saber.ula.ve/ bitstream/ handle/123456789/39304/perdomo_ flores_2014.pdf;jsessionid=A686A8901BFEC4C89DDDD4767F 78D225? sequence $=1$ [acceso 03/03/2018]

11. Blanco N, Roque Y, Betancourt Y, Ugarte Y, Reyes Y. Principales dificultades en los proyectos investigativos en residentes de las especialidades médicas. Edumecentro, [Internet] 2012; 4(1): 3946. URL disponible en: http://scielo.sld.cu/scielo. php?script=sci_arttext\&pid=S2077-28742012000100007 [acceso 08/04/2018]

12. Grasso L. Dificultades frecuentes en la elaboración de proyectos de trabajo de investigación y trabajos finales. Revista Tesis. [Internet] 2012;1:136-156. URL disponible en: https: / / revistas. unc.edu.ar / index.php / tesis / article / viewFile / 2880/ 2746 [acceso 07/06/2018] 